\title{
THE SCIENTIFIC WORK OF ELIAKIM HASTINGS MOORE
}

1. Introduction. In a previous number of this Bulletin* the writer of these pages has published a biographical sketch of the life of Eliakim Hastings Moore. No account of his life can approximate completeness, however, without a more detailed description of his scientific activities than was given there. His enthusiasm for mathematical research was a dominant one, more characteristic of him than any other, in spite of the fact that he had many administrative and editorial responsibilities which often interfered seriously with his scientific work. He had a catholic interest in all domains of mathematics and a breadth of knowledge which was remarkable. I have known few men with so great an appreciation of the mathematical efforts of others, or so well qualified to discuss them in many different fields, qualities which were an important part of his insignia of leadership. If there were two characteristics of his research which could be distinguished above others, I should say that they would be rigor and generality. He strove for precision in thought and language at a time when vagueness and uncertainty were common in mathematical literature, and he profoundly influenced both students and colleagues in this respect by his teaching and example. He was furthermore among the very first to recognize the possibility and importance of the great generality in analysis which is now sought by many writers.

Moore was a prolific thinker, though not throughout his lifetime a prolific writer. His papers, as given in the bibliography at the end of this article, fall roughly into the groups indicated in the following table which lists the numbers of the items in the bibliography belonging to each field and the dates of the first and last papers in each group:

I. Geometry; $1-4,28,41,43-44,47,63 ; 1885-1913$.

II. Groups, numbers, algebra; $6-9,12,13,15-18,20-27,29,32,33,42,46$, $48,53,60,68,69,71 ; 1892-1922$.

III. Theory of functions; $5,10,11,14,19,30,31,35-40,52,59,67,73,74$; 1890-1926.

IV. Integral equations, general analysis; $50,51,54,56,58,61,62,64-66$, $70 ; 1906-1922$.

V. Miscellaneous; 34, 45, 49, 55, 57, 72; 1900-1922.

The table indicates fairly well, I think, the sequence of his major interests, though it does not represent adequately the relative enthusiasms with which he pursued them. The domains suggested in the second and fourth entries were the ones to which he gave most thought. His studies in algebra and the theory of groups fell in the period of his greatest activity as a writer, while integral equations and general analysis were his absorbing interest during the latter part of his life when he published least. For general analysis, in particular, he never lost his enthusiasm. He continued his speculations in that field into the last year of his life, as long as his strength permitted.

* Vol. 39 (1933), pp. 831-838. 
The synopses of Moore's papers in the following pages are necessarily brief, but I hope that they may indicate clearly the development and sequence of his major interests. For analyses and evaluations of the papers in the first, second, and fourth groups of the table I am greatly indebted to my colleagues, Professors Logsdon, Dickson, and Barnard, respectively.

2. Geometry. Moore's papers on geometry fall for the most part into two groups, an early one concerned with algebraic geometry, and a later series of three papers on postulational foundations. In his doctoral dissertation (1)* of 1885 , written when he was 23 years old, Moore generalized to irreducible $r$ spreads of order $n$ three theorems of Clifford concerning skew curves. Such an $r$-spread always lies in a flat space of $n+r-1$ dimensions or less. If it lies in no space of fewer dimensions its points are in one-to-one correspondence with those of an $r$-spread of order $n-k+r+1$ in a flat space of $r+1$ dimensions, and it is unicursal. Furthermore every flat section of such an $r$-spread is also unicursal. In a second part of the paper Moore discusses 2-spreads of order $m$. By projecting such a surface on two planes he sets up a Cremona transformation between the planes, and uses this correspondence to obtain a canonical equation of the surface in $(m+1)$-space and properties of its osculating flat spaces and the spreads generated by them. The consideration of curves on the surface leads to a curvilinear generalization for them of Pascal's theorem concerning a hexagon, and to various generalizations of the theory of plane curves. In the latter part of the paper he generalizes to spreads of odd order on hyper-quadrics theorems which Cayley had deduced for super-lines of quadric surfaces in five dimensions.

The second paper (2) was a note, with C. N. Little, probably written before his dissertation but published in 1886, concerning the number and character of the regions into which a plane is divided by $n$ straight lines. Some special cases of the division of spaces of $k$ dimensions by flat spaces of $k-1$ dimensions were also discussed.

The paper (3) of 1888 is concerned with Picard's theorem stating that the only algebraic surfaces whose plane sections are unicursal are unicursal ruled surfaces and the surface of fourth order of Steiner. It is proved that such a surface may be regarded as the projection of a unicursal surface of the same order $N$ in a flat space of $N+1$ dimensions. Moore also arrives incidentally at the result, not formulated explicitly, that every complete linear system of plane curves of degree $n$ can be transformed, by a Cremona transformation, either into a system of curves of order $n$ possessing an $(n-1)$-fold base point and $n-1$ simple base points, or into the system of $\infty^{5}$ conics.

In the paper (4) of 1888 Moore studied the conditions for six points $K_{i}$, $(i=1, \cdots, 6)$, in a plane to form two curvilinearly perspective triangles in a net of conics. If the points $K_{i}$ and two of the base points of the net, $H_{1}$ and $H_{2}$, are selected arbitrarily, the third base point $H_{3}$ and the center $I$ of curvilinear perspectivity are corresponding points of an involution on a quartic curve which has $H_{1}$ and $H_{2}$ as double points and passes through the six points $K_{i}$. If the points $K_{i}$ form two perspective triangles in the ordinary straight line

* The numbers in parentheses refer to the bibliography at the end of this paper. 
sense, then the above-mentioned quartic reduces to the straight line $H_{1} H_{2}$ and a cubic of the pencil of cubics through the eight points $H_{1}, H_{2}, K_{i}$. If the points $K_{i}$ form more than one pair of rectilinearly perspective triangles, they will have corresponding curvilinear perspective properties in every net of conics having base points $H_{1}, H_{2}, H_{3}$ which with the points $K_{i}$ are the nine base points of a pencil of cubics, and conversely. By applying quadratic Cremona transformations to these configurations Moore finds necessary and sufficient conditions for six points to have curvilinear perspective properties analogous to those described above in nets of curves of order $2^{n}$.

The qualities exhibited by Moore in this early group of papers were in many ways characteristic of his research throughout his life. The theory of linear systems of plane curves, which he freely uses, was at that time a central interest in algebraic geometry, as indicated for example in numerous papers which appeared between 1884 and 1887 in the Palermo Rendiconti. The skill which Moore showed in handling such systems, and the elegance of his results, are indicative of unusual power in so young a man, and the problems which he studied were fundamental ones for the algebraic geometry of that period.

Moore published in 1900 one further paper (28) on algebraic geometry which might also be classed in Group II of the above table. The anharmonic ratios formed from $n$ variables $z_{1}, \cdots, z_{n}$ are all expressible rationally in terms of a fundamental system $r_{i}=\left(z_{n}, z_{n-1}, z_{n-2}, z_{i}\right),(i=1, \cdots, n-3)$, consisting of $n-3$ of them. The $n$ ! fundamental systems formed by permuting the variables $z_{i}$ are therefore all rationally expressible in terms of $\left(r_{1}, \cdots, r_{n-3}\right)$, and a group of $n$ ! Cremona transformations of the $(n-3)$-dimensional flat space of points $\left(r_{1}, \cdots, r_{n-3}\right)$ is thus defined. The group has as a sub-group Klein's group of $(n-1)$ ! collineations which permute a certain set of $n-1$ fixed points $P_{k},(k=1, \cdots, n-1)$, among themselves. The remaining transformations of the group are $n !-(n-1)$ ! Cremona transformations whose critical figures are included in the complete $(n-1)$-gon of points $P_{k} *$ For the Klein group mentioned above Moore found a fundamental region in the paper (32).

The appearance of Hilbert's book on the foundations of geometry in 1899 attracted the attention of Moore and his students to postulational methods, including the earlier work of Pasch and Peano as well as that of Hilbert. In his paper (41) of 1902, Moore gave a new formulation of a system of axioms for $n$-dimensional projective geometry, using points only as undefined elements instead of the points, lines, and planes of Hilbert in the 3-dimensional case, and defining $k$-spaces as classes of points with suitably postulated properties. Schur had asserted in 1901 that a certain three of Hilbert's axioms were provable from certain others. This statement Moore showed to be incorrect. He did, however, find that two of Hilbert's axioms are redundant. Moore's papers (43, 44) were also concerned with foundations, but were perhaps of lesser importance.

A final paper (47) which may be classed with the geometrical group con-

* A more detailed discussion for the case $n=5$ was given by H. E. Slaught in his dissertation, The cross-ratio group of 120 quadratic Cremona transformations of the plane, American Journal of Mathematics, vol. 22 (1900), pp. 343388. 
tains a proof that through every pair of points in the upper half-plane there passes one and but one of a two-parameter family of very general arches. This result is a generalization of a well known theorem concerning the cycloid arches of the brachistochrone problem in the calculus of variations.

3. Groups, Numbers, Algebra. Moore early became interested in the theory of abstract groups, one of the fields of research in which he was at various times most deeply engaged. An abstract of his first paper (6) in this field appeared in 1892 , but his first published paper was the paper $(8,16)$ of 1893 . If $q$ is a prime greater than 3 , the group of the modular equation for the transformation of elliptic functions of order $q$ is known to consist of all linear fractional transformations on one variable having integral coefficients, taken modulo $q$, of determinant unity. The generalization in the paper (8) is the case in which the coefficients are Galois imaginaries, that is, polynomials in $x$ taken with respect to the moduli $q, f(x)$ where $f(x)$ is an irreducible polynomial modulo $q$. Moore also proved in this paper the interesting and important theorem that every finite field is a Galois field. In the later paper (46) of 1903 he determined all of the sub-groups of his generalized modular group.

An isomorphism of an abstract group $G$ was defined by Moore in the paper (9) 1894 to be a substitution on the elements of $G$ which preserves the multiplication table of $G$. The resulting group of isomorphisms had been defined independently by $O$. Hölder.* Later, in the paper (13) of 1895, Moore took $G$ to be the Abelian group of order $p^{n}$ of type $(1,1, \cdots, 1)$, and proved that its group of isomorphisms is Jordan's group of all linear homogeneous transformations on $n$ variables whose coefficients are integers taken modulo a prime $p$. He defined three related tactical configurations. Elsewhere, in the paper (21) of 1897 , he defined abstract groups which are simply isomorphic with the general symmetric and alternating substitution groups. In the paper (23) of 1896 and 1898 Moore announced his discovery of the important fact that every finite group $G$ of linear transformations on $n$ variables has a Hermitian invariant. If one starts with any positive Hermitian form, applies to it all of the transformations of $G$, and adds the resulting forms, the sum is evidently invariant under $G$. This theorem was announced independently by A. Loewy and Fuchs.

One should mention here the interesting group of Cremona transformations in Moore's paper $(28,32)$ of 1900 , described in an earlier paragraph above. His definitions of an abstract group, in the papers $(42,48)$ of 1902 and 1905, are well known.

Several of his papers $(7,12,22,24)$ employ the notion of a triple system, that is, an arrangement of $n$ letters in triples (the order of the letters in a triple being immaterial) such that every pair appears exactly once in some triple. For $n=7$ the unique triple system of the ordered letters $a b c d r s t$ is $a b c, a d r$, $a s t, b d s, b r t, c d t, c r s$. As an application Moore studied in the paper (26) of 1899 the resolvent equation of degree 15 of the general equation of degree 7 by using the 30 equivalent triple systems in 7 letters (each invariant under a group of 168 even substitutions whose index under the alternating group is 15). Next he used a quadruple system in 8 letters such that every triple appears in one and

* Mathematische Annalen, vol. 43 (1893), p. 314. 
only one quadruple. There are 14 quadruples in the system. Each quadruple system is invariant under a group of $8 \times 168$ even substitutions whose index under the alternating group of order $8 ! / 2$ is 15 . Hence the general equation of degree 8 has a resolvent of degree 15 .

Finally Moore applied groups, in the paper (18) of 1896 , to a highly abstract theory which includes as special cases whist tournament arrangements, and various generalizations of the problem of 15 school girls involving a triple system with $n=15$.

Moore published also a series of papers concerning the theory of numbers and modular systems. In homogeneous form Fermat's theorem states that $x^{p} y-y^{p} x$ is identically congruent to a product of linear functions modulo a prime $p$. Moore proved, in the paper (17) of 1896, the corresponding theorem for the determinant

$$
\left|\begin{array}{lll}
x & y & z \\
x^{p} & y^{p} & z^{p} \\
x^{p^{2}} & y^{p^{2}} & z^{p^{2}}
\end{array}\right|
$$

and for the analogous determinant of order $n$. In the paper (53) of 1907 the modular system composed of the coefficients of the difference between the determinant and the product of linear functions modulo $p$ mentioned above was decomposed into prime modular systems. Elsewhere, in (20) of 1897, Moore filled a gap in a proof by Molk concerning general modular systems.

The papers $(29,15)$ of 1900 and 1896 were devoted to algebraic questions. In the former Moore discussed a determinant $D$ each of whose elements is a product of two factors, in particular, the factorization of $D$ into determinants. In the latter he denotes by $f(x, m)$ the function

$$
a+h(m+x)+b m x+g\left(m^{2}+x^{2}\right)+f\left(m^{2} x+x^{2} m\right)+c m^{2} x^{2}
$$

symmetric in $m, x$ and of degree 2 in each. Let $x_{1}, x_{2}$ be the roots of $f=0$. Then $f\left(x_{1}, x_{2}\right)$ is zero identically in $m$ if and only if $(b-g) g+a c-f h=0$, except when $f$ is $g(x-m)^{2}$.

4. Theory of Functions. Moore's interest in the theory of functions was first indicated in a paper (5) of 1890 concerning elliptic functions, and a second paper (11) of 1895 concerning the characteristics of theta-functions. They were a completion and revision of proofs of theorems by Halphen and Prym, respectively. A much more important contribution, showing perhaps for the first time his full power in analysis, was his memoir (19) of 1896 concerning transcendentally transcendental functions, inspired by Hölder's proof that the function $\Gamma(x)$ satisfies no algebraic differential equation with coefficients rational in the variable $x$. Moore defines a "realm of rationality" as the totality of rational functions of $n$ analytic functions $f_{i}(x)(i=1, \cdots, n)$ having a common domain of existence, the coefficients in the rational functions being complex constants. A function $\phi(x)$ is transcendentally transcendental with respect to such a realm if it satisfies no algebraic differential equation with coefficients in the realm. Moore established an ingenious sufficient condition that a function satisfying a functional equation of very general type shall be transcendentally transcendental, and applied it to show that this property is possessed by the two functions 


$$
\phi(x)=\sum_{\nu=0}^{\infty} x^{a^{\nu}}, \quad \psi(y)=\sum_{\nu=0}^{\infty} e^{a^{\nu} y} \quad(a=\text { integer }>1),
$$

defined for $|x|<1$ and real part of $y$ negative and satisfying the functional equations $\phi\left(x^{a}\right)=\phi(x)-x$ and $\psi(a y)=\psi(y)-e^{y}$. In the latter part of the paper he applies his methods to prove that every solution of the functional equation $\Gamma(x+1)=x \Gamma(x)$ is transcendentally transcendental in the realm of rationality of the single function $f_{1}(x)=x$.

In 1900 mathematicians in this country were greatly interested in Goursat's recapitulation, in the first volume of the Transactions of the American Mathematical Society, of his proof of Cauchy's theorem for a function $f(z)$ without the assumption of the continuity of the derivative $f^{\prime}(z)$. In a paper (31) of the same year Moore exhibits a proof of this important result by an indirect method which seems unusually useful and effective.

Weierstrass described a continuous plane curve possessing nowhere a tangent, and Peano and Hilbert have given examples of continuous plane curves $x=\phi(t), y=\psi(t)$ which completely cover portions of the plane. In his paper (30) of 1900 Moore re-studies in illuminating geometrical-analytical fashion the curves of Peano and Hilbert. He re-defines geometrically the $t y$-curve $y=\psi(t)$ of Peano and shows that it also is an example of a continuous curve possessing nowhere a well-defined tangent. It is in some ways a more interesting example than that of Weierstrass since it is possible to show that the curve has nowhere a progressive or regressive non-vertical tangent, and to characterize the points, everywhere dense on the curve, at which it has progressive or regressive vertical tangents.

In 1901 Moore published three papers $(35,36,37)$ on improper definite integrals. They were concerned especially with theories of Harnack, Stolz, Jordan, Hölder, Schoenfliess, and de la Vallée Poussin, which occupied a dominant position in the theory of integration up to the time of the development of the newer theories of Borel, Lebesgue, and their followers. As the basis of his theory Moore adopted and improved notions of Harnack. Let $\Xi$ be a closed point set of Jordan measure zero on a finite interval $a \leqq x \leqq b$, and let $F(x)$ be a real single-valued function at each point of $a b$ not in $\Xi$. Let $I$ be a set of a finite number of discrete intervals containing the points of $\Xi$ as interior points, and let $F_{I}(x)$ be a function equal to $F(x)$ outside of $I$ and equal to zero on $I$. Then the $\Xi$-integral of $F(x)$ on $a b$

$$
\int_{a \Xi}^{b} F(x) d x
$$

is said to exist if the Riemann integral of $F_{I}(x)$ exists on $a b$ for every $I$ and approaches a finite limit as the measure of $I$ approaches zero. There are two kinds of $\Xi$-integrals, the "narrow" and the "broad", according as every interval of the set $I$ is required to contain at least one point of $\Xi$ or not. The two types have differences analogous to those of conditional and absolute convergence. In his first paper (35) Moore analyzed these differences and gave a masterly discussion of Harnack integrals and their relations to the improper integrals of other writers. Preceding memoirs contained a number of important theorems whose truth had been doubted or falsely asserted. In characteristic fashion Moore established by proofs the ones which were true and constructed ex- 
amples showing the failure of the others. His second paper (36) in this field was an outgrowth of the first. It contains a classification of the improper integrals of preceding writers and a definition by postulational methods of a new and more general type including the others as special cases. The paper (37) is concerned with two integrability theorems of Du Bois-Reymond. The first states that a continuous function of integrable functions is integrable, and the second that an integrable function of integrable functions is integrable. Moore generalizes the former of these theorems and gives an example to show that the latter is incorrect. Professor Oswald Veblen has recently remarked to me that the relative effectiveness of the integration theory of Borel and Lebesgue, and other equivalent theories, is undoubtedly due to the presence of the two limiting processes which they utilize, a first in the definition of measure and a second in the subsequent definition of the integral. It is evident, even from the brief description above given, that the former of these limiting processes is absent in the older theories in which Moore and many others were interested.

The paper (52) is a note explaining in interesting fashion how the Fourier constants of a product $f(x) g(x)$ can be determined from those of $f(x)$ and $g(x)$ by a calculation involving as its principal step the formal multiplication of two Laurent series.

The paper (73) on a general theory of limits by E. H. Moore and H. L. Smith, published in 1922, should perhaps be classed with the papers on general analysis, but it has great interest for students of the theory of functions in general. In his general analysis theories Moore had introduced the following notion of a limit. Let $Q$ be a class of elements $q$ and $S$ the class of all finite classes $s$ of elements $q$. A numerically valued function $\alpha(s)$ on the range $S$ is said to converge to a number $a$ as a limit if for every $e>0$ there exists a class $s_{e}$ such that $|\alpha(s)-a|<e$ for every class $s$ containing $s_{e}$. The paper here under discussion gives a definition of a generalized limit which includes the ordinary limit of a sequence and the limit just described, as well as many other conceivable cases, as special instances. Let $P$ be a class of elements $p$ and $R$ a relation such that for every pair of elements $p_{1}, p_{2}$ of $P$ the element $p_{1}$ is either in the relation $R$ to $p_{2}$ or not. Let $\alpha(p)$ be a numerically valued function on the range $P$. Then $\alpha(p)$ has by definition the limit $a$ provided that for every $e>0$ there exists an element $p_{e}$ such that $|\alpha(p)-a|<e$ for every $p$ in the relation $R$ to $p_{e}$, in notation $p R p_{e}$. The relation $R$ is supposed to be transitive, so that $p_{1} R p_{2}$ and $p_{2} R p_{3}$ imply $p_{1} R p_{3}$, and it has the composition property that for every pair of elements $p_{1}, p_{2}$ there exists an element $p_{3}$ such that $p_{3} R p_{1}$ and $p_{3} \quad R \quad p_{2}$. The paper contains a thorough analysis of the properties of these limits including such topics as uniform convergence, double limits, and a revised formulation of theorems of Fréchet concerning compact sets and covering theorems.

5. Integral Equations, General Analysis. Moore's interest in the postulational foundations of various mathematical theories was indicated as early as 1893 , in his paper $(8,16)$ in which he set down simple postulates for an abstract field and showed that every such field is the abstract form of a Galois field. It was an interest which appeared frequently in his papers, however, notably in his characterization (36) of a generalized type of improper integral, in his papers $(42-44)$ on the foundations of geometry, in his definitions $(41,48)$ 
of an abstract group, and in his presidential address (45) on the foundations of mathematics. In the early years of this century, when various theories of integral equations followed one another rapidly, his attention was led quite naturally, in accord with this interest in foundations, to the formulation of postulational theories which should include as special instances numerous known theories of linear equations. His guiding principle, as often stated, was that "the existence of analogies between central features of various theories implies the existence of a general abstract theory which includes the particular theories and unifies them with respect to those central features."

Moore found that the special theories of linear equations which he desired to unify could be regarded as special instances of a general theory of linear functional equations in which the functions $\mu(p)$ involved are defined on an entirely unrestricted range $P$ of elements $p$. In his efforts to preserve the generality necessary in this range he soon discovered that it was impractical to try to generalize such properties as continuity or differentiability of functions. He was able to leave the range quite unrestricted, however, if he presupposed that the functions $\mu$ with which he was working belonged to a class $\mathfrak{M}$ of such functions, and if he postulated suitable properties for the class $\mathfrak{M}$ as a whole. This idea was effective in generalizing the theory of linear equations, and it seemed to promise similar extensions in many other domains of mathematics. Thus Moore was led to the comprehensive development of the theory of classes of functions on a general range which he called "general analysis".

The principle of generalization quoted above was the dominant note of Moore's colloquium lectures at Yale University in 1906, but his paper (54), presented at the International Congress of Mathematicians in Rome in 1909, was the first detailed publication indicating the form which the theory of general analysis was taking in his mind. The functions $\mu$ which he studied in this paper have numerical values $\mu(p)$ defined for an entirely arbitrary range $\mathrm{P}$ of elements $p$. The class $\mathfrak{M}$ to which they belong possesses, besides the usual linearity properties, the so-called properties of dominance and self-closure, and a composition property. Functional transformations between classes of functions are considered, and various properties of these transformations, such as linearity, boundedness, and norm properties, are introduced. The result was a general theory of transformations which included the transformations of the special cases of linear equation theory from which Moore started, and of course much more besides. Instances of the general theory are the class $\mathfrak{M}$ of Hilbert sequences regarded as functions of $p$ on the range of elements $p=0,1,2, \cdots$, the class of continuous functions on the range $0 \leqq p \leqq 1$, and their transformations. The success of Moore's theory in including such heterogeneous special cases was dependent largely upon his notion of uniform convergence with respect to a scale-function, which was frequently effective in his work, and which will be described somewhat further in a later paragraph of this paper. The paper closes with a study of the linear functional differential equation

$$
\partial \rho(t, p) / \partial t=K \boldsymbol{\rho}, \quad\left(t_{1} \leqq t \leqq t_{2} ; p \text { in } \mathfrak{P}\right)
$$

where $K$ is a linear functional transformation taking $\rho(t, p)$ into a function of the same arguments. The differential equation with suitable initial conditions is equivalent to a linear integral equation which has a unique solution. 
The memoir (56), published in 1910, is a systematic introduction to the form of general analysis, which, as indicated above, was inaugurated in Moore's colloquium lectures of 1906. The first part of the lectures is devoted to a study of the closure and dominance properties of classes $\mathfrak{M}$ of real single-valued functions $\mu$ on a general range $P$ of elements $p$. Much use is made of the important notion of uniform convergence of a sequence $\mu_{n},(n=1,2, \cdots)$, to a function $\mu$ relative to a scale function $\sigma$. Such convergence means that for every $e>0$ there exists an integer $n_{e}$ such that $\left|\mu_{n}(p)-\mu(p)\right|<e \sigma(p)$ for every $n>n_{e}$ and $p$ in $\mathrm{P}$. The second part of the paper is concerned with properties of a class of functions of two variables on two general ranges, the class being obtained by various extensions of the class of products of pairs of functions from two of the classes considered in the first part of the paper. Desirable properties of the product class may be obtained by imposing suitable properties on one or both of the component classes.

In two papers $(58,61)$ of the years 1912 and 1913, respectively, Moore gave in essential outline the Fredholm theory of linear integral equations, and the Hilbert-Schmidt theory of integral equations with Hermitian kernels, from the point of view of his general analysis. In the former of these papers he notes that an obvious basis for the Fredholm theory, in notation ( $\mathfrak{A} ; \mathrm{P} ; \mathfrak{M} ; \mathfrak{R} ; J)$, consists of the class $\mathfrak{A}$ of real numbers, a range $P$ of undefined elements $p$, a class $\mathfrak{M}$ of functions $\mu$ on $\mathrm{P}$ to $\mathfrak{A}$, a class $\Re$ of kernel functions $\kappa$ on pairs $\left(p^{\prime}, p^{\prime \prime}\right)$ to $\mathfrak{A}$, and a linear functional operation $J$. The functional equation studied has the form $\xi=\eta-z J \kappa \eta$, where $z$ is a real parameter, $\xi$ and $\kappa$ are given functions in their respective classes $\mathfrak{M}$ and $\Re$, and a solution $\eta$ is to be found in $\mathfrak{M}$. By means of the developments in his previous paper (56) he successively finds modified or simplified bases, altogether six in number, with respect to which the theory can be carried through. It is interesting and important to note that on account of the flexibility of the range $P$ the theory of a system of linear equations for functions $\eta$ of several variables is included in the theory of a single equation for a single function $\eta$ of a single variable $p$. The paper (61) of 1912 is concerned especially with results analogous to those of the Hilbert-Schmidt theory of linear integral equations with symmetric or Hermitian kernel functions $\kappa$. Very general results are found by means of a theory of linear Hermitian positive definite functional operations. As has been remarked by Professor T. H. Hildebrandt, ${ }^{*}$ the methods of Moore in the theory of linear functional equations are epoch-making in that they shift the attention from the properties of individual functions to properties of classes of functions, and from the form of the operator $J$ to its properties, thus attaining far-reaching generality.

The characteristic value problem in the theory of linear equations involving a denumerable infinity of variables, as presented for example by Hilbert, gave rise to results which Moore failed to attain in the general theory which he had developed in the papers described in the preceding paragraphs. Attempts to modify his theory led to such complexities that he finally abandoned the highly postulational method of attack in favor of a constructive theory. The results which he attained are in their final formulation concerned only with a

* In his address at the memorial meeting of the American Mathematical Society in honor of E. H. Moore, Chicago, April 14, 1933. 
positive Hermitian matrix function $\epsilon$ of pairs of arguments $\left(p^{\prime}, p^{\prime \prime}\right)$ on the same range $\mathrm{P}$, and with suitable definitions of an integration process $J$ and a class of integrable functions. In terms of these notions he developed in the years following 1915 a theory of integral equations, characteristic values, and expansions, which is a generalization of the analogous theory for the limited matrices of Hilbert. The details of this work of Moore have remained for the most part unpublished, but are now being assembled and will appear in print. The limiting process which was used in defining his integral was first described in the paper (65) of 1915 , but was afterward presented in more detail in the joint paper (73) with H. L. Smith published in 1922 and described in a preceding paragraph. The paper (70) of 1922 , on power series in general analysis, gives the most important results of a chapter in the new theory which may be roughly described as a generalized Fourier series theory. The first part of the paper gives an illustration of these results by means of an application to certain types of power series in which the number of variables is not necessarily denumerably infinite.

In the bibliography at the end of this paper a number of abstracts $(50,51$, $62,64,66)$ are listed which were concerned with general analysis. Of these the first four have to do with Moore's earlier theory, and the contents of the papers they describe are either included in his more extensive memoirs, or are associated with parts of the theory in a readily understandable way. The last (66) is the title only of an address by Moore in 1915 as chairman of the Chicago Section of the American Mathematical Society. It undoubtedly concerned the second theory of general analysis which was then taking form in his mind, but we have not been able so far to find a record of it other than what may be contained in the manuscripts of the theory now being prepared for publication.

In concluding these remarks concerning Moore's theories of general analysis I wish to call attention to a sentence from the address of Professor E. W. Chittenden at the memorial meeting referred to on a preceding page: "The justification for general analysis and similar general theories will not be found in the contributions which are made to the special theories which suggest the generalization. Nevertheless, minor contributions do result from the methods of approach required by the general point of view. The desired justification lies in the contribution of the general theory to a more perfect comprehension of the nature and significance of the underlying mathematical elements, in the resulting clarification and condensation of proof, and in the extension of the range of application for a significant group of ideas".

6. Miscellaneous Papers. The titles of the papers in the group designated as miscellaneous in the table are for the most part self-explanatory. Three of these should be mentioned more explicitly, however, Moore's addresses (45, 55,72 ) as retiring president of the American Mathematical Society in 1902, at the 20th anniversary of Clark University in 1909, and as retiring president of the American Association for the Advancement of Science in 1922. The first contains in its earlier pages an illuminating description of Moore's conception of the logical structures of pure and applied mathematical sciences, the latter part being devoted to a discussion of the pedagogical methods by means of which one might hope to establish such concepts clearly in the minds of students in our schools, colleges, and universities. It was written at a time when 
Moore himself was greatly interested in a laboratory method of instruction for college students of mathematics, and at the height of the so-called Perry movement in England which aroused great interest and discussion among those responsible for instruction in the mathematical sciences in our own country.

The second paper (55) was apparently unpublished and we have as yet found no manuscript. But there is a somewhat informally written paper with nearly the same title and date in the archives of the Department of Mathematics at the University of Chicago. There seems little doubt that it contains the material of the Clark address. It contains a non-technical description of the work of Pasch, Peano, and Hilbert on foundations of geometry, and of the contributions of Cantor, Russell, and Zermelo to the theory of classes.

The address (72) as retiring president of the American Association was also unpublished, but a type-written copy is extant. It is a description of the historical development of the number systems of mathematics with the purpose of establishing the interesting thesis that mathematical theories, though well recognized as highly deductive in their ultimately sophisticated forms, are nevertheless the products of inductive developments similar to those well known in the laboratory sciences.

Moore presented numerous papers before the American Mathematical Society whose contents did not afterward appear in print or which appeared under different titles. The very interesting notions which he had concerning double limits in the paper (38), for example, were developed in Chapter IX of his lectures on "Advanced Integral Calculus" in 1900, a hand-written account of which by Professor Oswald Veblen is in the University of Chicago library. The material described in the abstract (67) seems to be included in somewhat modified form in the published paper (73), and (74) is a part of Moore's second theory of general analysis now in preparation for publication.

\section{BIBLIOGRAPHY}

The abbreviations used are explained at the end of the bibliography. Items marked with an asterisk are titles of more significant abstracts or unpublished addresses.

1. Extensions of certain theorems of Clifford and Cayley in the geometry of $n$ dimensions, Transactions of the Connecticut Academy of Arts and Sciences, vol. 7 (1885), pp. 1-18.

2. Note on space divisions (with C. N. Little), Journal, vol. 8 (1886), pp. 127131.

3. Algebraic surfaces of which every plane section is unicursal in the light of n-dimensional geometry, Journal, vol. 10 (1888), pp. 17-28.

4. A problem suggested in the geometry of nets of curves and applied to the theory of six points having multiply perspective relations, Journal, vol. 10 (1888), pp. 243-257.

5. Note concerning a fundamental theorem of elliptic functions, as treated in Halphen's Traité, vol. 1, pp. 39-41, Rendiconti del Circolo Matematico di Palermo, vol. 4 (1890), pp. 186-194.

*6. Concerning a congruence group of order 360 contained in the group of linear fractional substitutions, Proceedings of the American Association for the Advancement of Science, vol. 41 (1892), p. 62. 
7. Concerning triple systems, Annalen, vol. 43 (1893), pp. 271-285.

*8. A doubly-infinite system of simple groups, Bulletin of the New York Mathematical Society, vol. 3 (1893), pp. 73-78; see also No. 16 below.

9. The group of holoedric transformations into itself of a given group, Bulletin, vol. 1 (1894), pp. 61-66.

10. Concerning the definition by a system of functional properties of the function $f(z)=(\sin \pi z) / \pi$, Annals, (1), vol. 9 (1894), pp. 43-49.

11. On a theorem concerning p-rowed characteristics with denominator 2 , Bulle tin, vol. 1 (1895), pp. 252-255.

12. Concerning triple systems, Rendiconti del Circolo Matematico di Palermo, vol. 9 (1895), p. 86.

13. Concerning Jordan's linear groups, Bulletin, vol. 2 (1895), pp. 33-43.

14. A note on mean values, American Mathematical Monthly, vol. 2 (1895), p. 303.

15. On an interesting system of quadratic equations (with Emma C. Acker mann), American Mathematical Monthly, vol. 3 (1896), p. 38.

16. A doubly-infinite system of simple groups, Mathematical Papers Read at the International Mathematical Congress in Chicago 1893, published by MacMillan, 1896, pp. 208-242; see also the abstract, No. 8 above.

17. A two-fold generalization of Fermat's theorem, Bulletin, vol. 2 (1896), pp. 189-199.

18. Tactical Memoranda, I-III, Journal, vol. 18 (1896), pp. 264-303.

19. Concerning transcendentally transcendental functions, Annalen, vol. 48 (1897), pp. 49-74.

20. The decomposition of modular systems of rank $n$ in $n$ variables, Bulletin, vol. 3 (1897), pp. 372-380.

21. Concerning the abstract groups of order $k !$ and $(k / 2) !$ holoedrically isomorphic with the symmetric and the alternating substitution groups on $k$ letters, Proceedings of the London Mathematical Society, vol. 28 (1897), pp. 357366.

22. Concerning regular triple systems, Bulletin, vol. 4 (1897), pp. 11-16.

23. An universal invariant for finite groups of linear substitutions with application in the theory of the canonical form of the linear substitution of finite period, Annalen, vol. 50 (1898), pp. 213-219.

24. Concerning abelian-regular transitive triple systems, Annalen, vol. 50 (1898), pp. 225-240.

*25. A two-parameter class of solvable quintics, in which the rational relations amongst the roots, by threes, do not contain the parameters, Bulletin, vol. 4 (1898), p. 364.

26. Concerning the general equations of the seventh and eighth degrees, Annalen, vol. 51 (1899), pp. 417-444.

*27. On the sub-groups of the Abelian groups, Bulletin, vol. 5 (1899), pp. 382 383.

28. The cross ratio group of $n$ ! Cremona transformations of order $n-3$ in flat space of $n-3$ dimensions, Journal, vol. 22 (1900), pp. 279-291.

29. A fundamental remark concerning determinantal notation and the evaluation of an important determinant of special form, Annals, (2), vol. 1 (1900), pp. 177188. 
30. On certain crinkly curves, Transactions, vol. 1 (1900), pp. 72-90.

31. A simple proof of the fundamental Cauchy-Goursat theorem, Transactions, vol. 1 (1900), pp. 499-506.

32. Concerning Klein's group of $(n+1)$ ! n-ary collineations, Journal, vol. 22 (1900), pp. 336-342.

*33. On the generational determination of abstract groups, Bulletin, vol. 6 (1900), pp. 379-380.

34. The undergraduate curriculum (E. H. Moore et al.), Bulletin, vol. 7 (1900), pp. 14-24.

35. Concerning Harnack's theory of improper definite integrals, Transactions, vol. 2 (1901), pp. 296-330.

36. On the theory of improper definite integrals, Transactions, vol. 2 (1901), pp. 459-475.

37. Concerning Du Bois-Reymond's two relative integrability theorems, Annals, (2), vol. 2 (1901), pp. 153-158.

*38. On the uniformity of continuity, Bulletin, vol. 7 (1901), p. 245.

*39. On double limits, Bulletin, vol. 7 (1901), p. 257.

*40. Concerning the second mean-value theorem of the integral calculus, Bulletin, vol. 8 (1901), pp. 19-20.

41. On the projective axioms of geometry, Transactions, vol. 3 (1902), pp. 142158.

42. A definition of abstract groups, Transactions, vol. 3 (1902), pp. 485-492.

43. The betweenness assumptions, American Mathematical Monthly, vol. 9 (1902), pp. 152-153.

*44. On Hilbert's plane arguesian geometry, Bulletin, vol. 8 (1902), p. 202.

45. On the foundations of mathematics (retiring address as president of the American Mathematical Society), Bulletin, vol. 9 (1903), pp. 402-424; also in Science, (2), vol. 17 (1903), pp. 401-416.

46. The subgroups of the generalized finite modular group, The Decennial Publications of the University of Chicago (1903), pp. 141-190.

47. On doubly infinite systems of directly similar convex arches with common base line, Bulletin, vol. 10 (1904), pp. 337-341.

48. On a definition of abstract groups, Transactions, vol. 6 (1905), pp. 179-180.

49. The cross-section paper as a mathematical instrument, School Science and Mathematics (1906), pp. 429-450; also in The School Review, vol. 14 (1906), pp. 317-338.

*50. On the theory of systems of integral equations of the second kind, Bulletin, vol. 12 (1906), p. 280.

*51. Homogeneous distributive functional operations of degree $n$, Bulletin, vol. 13 (1907), pp. 217-219.

52. Note on Fourier's constants, Bulletin, vol. 13 (1907), pp. 232-234.

53. The decomposition of modular systems connected with the doubly generalized Fermat theorem, Bulletin, vol. 13 (1907), pp. 280-288.

54. On a form of general analysis with application to linear differential and integral equations, Atti del IV Congresso Internazionale dei Matematici (Rome, 6-11 Aprile, 1908), vol. 2 (1909), pp. 98-114.

*55. The role of postulational methods in mathematics (address at Clark University, 20th anniversary), Bulletin, vol. 16 (1909), p. 41. 
56. Introduction to a form of general analysis, New Haven Mathematical Colloquium, Yale University Press (1910), pp. 1-150.

57. A generalization of the game called Nim, Annals, (2), vol. 11 (1910), pp. 93-94.

58. On the foundations of the theory of linear integral equations, Bulletin, vol. 18 (1912), pp. 334-362.

*59. Multiplicative interrelations of certain classes of sequences of positive terms, Bulletin, vol. 18 (1912), pp. 444-445.

60. A mode of composition of positive quadratic forms, British Association Report, Dundee, vol. 82 (1913), p. 413.

61. On the fundamental functional operation of a general theory of linear integral equations, Proceedings of the Fifth International Mathematical Congress (Cambridge, 1912), vol. 1 (1913), pp. 230-255.

*62. On nowhere negative kernels, Bulletin, vol. 19 (1913), pp. 287-288.

*63. On the geometry of linear homogeneous transformations of $m$ variables, Bulletin, vol. 19 (1913), pp. 457-458.

*64. On a class of continuous functional operations associated with the class of continuous functions on a finite linear interval, Bulletin, vol. 20 (1913), pp. 70-71.

65. Definition of limit in general integral analysis, Proceedings of the National Academy of Sciences, vol. 1 (1915), pp. 628-632.

*66. Report on integral equations in general analysis (address by chairman of Chicago Section), Bulletin, vol. 21 (1915), p. 430.

*67. On a definition of the concept: limit of a function, Bulletin, vol. 22 (1916), pp. 439-440.

*68. On properly positive Hermitian matrices, Bulletin, vol. 23 (1916), pp. 6667.

*69. On the reciprocal of the general algebraic matrix, Bulletin, vol. 26 (1920), pp. 394-395.

70. On power series in general analysis, Annalen, vol. 86 (1922), pp. 30-39.

*71. On the determinant of a Hermitian matrix of quaternionic elements, Bulletin, vol. 28 (1922), p. 296.

*72. What is a number system?, Bulletin, vol. 29 (1922), p. 91.

73. A general theory of limits (with H. L. Smith), Journal, vol. 44 (1922), pp. 102-121.

*74. Introduction to a theory of generalized Hellinger integrals, Bulletin, vol. 32 (1926), p. 224.

\section{List of AbBreviations}

Annalen $=$ Mathematische Annalen

Annals = Annals of Mathematics

Bulletin $=$ Bulletin of the American Mathematical Society

Journal = American Journal of Mathematics

Transactions $=$ Transactions of the American Mathematical Society

G. A. Bliss 